\title{
Análise do comportamento e a habilidade de leitura: um levantamento crítico de artigos do JABA
}

\author{
Luiza Cristina Manad \\ Maria do Carmo Guedes \\ Roberta Gurgel Azzi
}

\begin{abstract}
Resumo
O presente trabalho pretendeu, por meio de uma análise de revisão de artigos sobre habilidade de leitura no ensino regular no Journal of Applied Behavior Analysis - JABA nos anos de 1968 a 2002, identificar as formas de atuação propostas pela Análise do Comportamento para ensinar ou remediar esta habilidade. Montando-se um banco de dados de artigos encontrados e utilizando-se palavras-chave que nortearam a busca na web site do periódico, foi possível identificar os delineamentos experimentais utilizados nas intervenções, as variáveis experimentais manipuladas pelo experimentador, as variáveis de observação que foram tanto um comportamento apresentado pelo sujeito quanto uma modificação ambiental expressando esse comportamento, os tipos de reforçadores empregados e o objetivo de cada intervenção. O espectro das variáveis analisadas indicou múltiplas possibilidades interventivas que podem ser aplicadas em uma sala de aula do ensino regular de leitura aumentando a performance do aluno nesta habilidade. Na condição de prática do professor em procedimentos operantes pode ser possível aumentar a performance de ler do aluno e os procedimentos de tutoria apresentados mostraram-se como alternativa efetiva para classes numerosas, o que se aproxima da realidade do ensino regular.

Palavras-Chave: Análise do comportamento; Habilidade de leitura; Alfabetização; Ensino regular.
\end{abstract}

\section{Behavior analysis and reading skills: A critical survey of JABA's articles}

\begin{abstract}
The present work intended to identify the approach ways proposed by the Behavior Analysis to teach or remedy this skill, throughout an analysis of an article review from the Journal of Applied Behavior Analysis - JABA's over the years 1968 to 2002 about reading skills on the regular teaching. A data bank was build up from an article selection found by using the key words that nurtured the search on the journal web site. It was possible to identify among the analysis data collected experimental design used on the interventions, experimental variables manipulated by the researcher; observational variables that were sometimes a behavior presented by the individual or, sometimes, an environment modification expressing this behavior, types of reinforcement used, and the main goal of each intervention. The experimental variables spectrum indicates multiple intervention possibilities, which can be applied on this academic teaching in a regular teaching class environment. Teacher practicing condition on the operant procedures makes possible to raise pupils reading performance, and the presented tutorial procedures showed as an effective alternative for big classes, what comes closer to the regular teaching reality.

Keywords: Behavior analysis; Reading skill; Reading and writing teaching; Regular teaching.
\end{abstract}

As questões educacionais têm sido objeto de estudo para as mais diferentes abordagens psicológicas. A publicação do livro organizado por Placco (2002), no qual é feita uma discussão sobre as contribuições da Psicologia para a Educação nos últimos 30 anos em diferentes concepções teóricas, evidencia essa pluralidade. Teóricos como Piaget, Vigotski, Rogers, Freud, Wallon e Skinner são apresentados pelos autores dos textos como iniciadores de um pensar singular sobre a Educação e, nesse contexto, a proposta de Skinner é abordada por Luna (2002), o qual ressalta que, embora a educação não tenha sido uma das áreas enfocadas sistematicamente por aquele autor enquanto tema de análise, é preciso considerar: a) as bases que estabelecen ao estender sua filosofia aos problemas educacionais e b) as interpretações que fez dos problemas educacionais a partir dos princípios estabelecidos pela pesquisa em análise experimental do comportamento nesse caso compondo aquela que veio a ser chamada análise do comportamento. (p. 146).

Um breve resgate de algumas passagens do pensamento de Skinner a respeito da contribuição da Análise do Comportamento para a educação exemplifica as assertivas de Luna mencionadas anteriormente e as apresenta como um aporte fundamental para a compreensão dos fenômenos educacionais.

${ }^{1}$ Endereço para correspondência:

Universitas - Centro Universitário de Itajubá-MG - R. Dr. Antonio Braga Filho, 687 - Caixa Postal 17- Itajubá-MG - 37501-002 
Em sua produção de mais de vinte e cinco artigos ou capítulos sobre o tema educação, Skinner apresenta a possibilidade de aplicação dos princípios básicos da Análise do Comportamento na área acadêmica e já em 1972 propunha uma melhoria do ensino por meio da aplicação de uma tecnologia:

Um ramo especial da psicologia, a assim chamada análise experimental do comportamento produziu, senão uma arte, pelo menos uma tecnologia do ensino, da qual se pode, com efeito, deduzir programas, esquemas e métodos de instrução. (p. 57)

Na mesma direção, ao propor o planejamento de contingências que promovem aquisição e fortalecimento de repertórios necessários ao desempenho acadêmico e que são úteis por toda a vida do aluno, Skinner (1974) coloca a Análise do Comportamento diante da expressão do objetivo da educação:

\section{O objetivo da educação pode ser expresso em termos compor- tamentais: um professor planeja contingências nas quais o aluno adquirirá comportamento que lhe será útil mais tarde, em outras contingências. As contingências instrutivas deverão ser planejadas. Não bá outra solução. (p. 158)}

Apresenta assim, uma alternativa às questões educacionais, trazendo-as para uma possibilidade de previsão, controle e análise, diferentemente das concepções que atribuem o objetivo da educação a razões outras que não as previsíveis e controláveis por encontrarem-se no meio ambiente.

A observação da rotina de uma sala de aula do ensino regular mostra a instalação e manutenção de repertórios de uma maneira que pouco considera a importância dos reforçadores, da correção imediata e da instalação por aproximações sucessivas do comportamento final desejado. Skinner (1972), comparando sua proposta de uma tecnologia de ensino com o ensino em sala de aula, comenta:

\section{É um grande choque passar desse excitante prospecto de uma ciência progressista do ensino para o ramo mais diretamente ligado ao processo de aprendizagem - a educação. (p. 13)}

Fazendo considerações sobre o ensino de aritmética, Skinner (1972) aponta as dificuldades do ensino regular em disponibilizar contingências que garantam o sucesso do aluno:

Talvez a mais séria crítica a sala de aula comum seja apontar a pouca freqüência do reforço. Uma vez que o aluno depende da professora para saber se está certo, e como muitos alunos em geral dependem da mesma professora, o número total de contingências que podem ser ordenadas durante, digamos os quatro primeiros anos, é da ordem de só alguns milhares. Entretanto, mesmo uma estimativa aproximada indica que o comportamento matemático eficiente nesse nível requer pelo menos algo da ordem de 25.000 contingências. (p. 16)

Esse hiato criado entre a prática cotidiana em sala de aula e as condições ótimas de uma situação educacional abre um espaço que pode ser ocupado pela análise do comportamento, pois a abordagem da educação nesta perspectiva possibilita uma postura científica na maneira de ensinar. Isso é confirmado por Vargas e Vargas (1992), que, ao fazerem um resgate do legado de Skinner para a educação, comentam a permanência de algumas características de sua proposta no ensino regular durante algumas décadas, considerando a assertiva:

\section{[...] sobretudo a educação tradicional adotou algumas características da instrução programada - objetivos comportamentais (chamados por uma variedade de nomes, na maioria das vezes de objetivos de performance); $e$ promoveu outros - reforçamento imediato (feedback). Durante os anos sessenta nos Estados Unidos, raras foram as escolas de ensino tradicional, com classes regulares, onde não foram aplicados alguns dos métodos propostos pela Análise do Comportamento. A utilização de características da instrução programada, da explicitação de objetivos em termos comportamentais ou a utilização de conseqüência imediata, estiveram presentes naquela década. Também a especificação completa do comportamento que se desejava ensinar e passos sucessivos para alcançá-lo foram largamente empregados nas escolas de educação de ensino regular em algumas áreas, como educação de pais, educação física e esportes. (p. 51)}

Outros autores, analistas do comportamento, como Holland e Keller, desenvolveram e publicaram trabalhos inovadores da área de educação, como a Instrução Programada, e o PSI, nos quais o planejamento das contingências de ensino garantiam a aquisição das habilidades para as quais foram planejadas. Keller (1983) conseguiu resultados por ele considerados satisfatórios com o PSI. Neste programa de ensino, cada aluno progredia em seu próprio ritmo e havia uma grande ênfase na participação deste aluno nas atividades propostas. As aulas expositivas puderam ser reduzidas ao mínimo enquanto instrumento de ensino e:

[...] contingências reforçadoras úteis na modelagem de habilidades simples como as de um operador de rádio, podem ser usadas também para desenvolver repertórios

Psico-USF, v. 9, n. 1, p. 59-69, Jan./Jun. 2004 
verbais, comportamentos conceituais e técnicas de laboratório, no contexto da educação universitária. (p. 130)

O embasamento experimental da proposta é a referência apresentada por Bijou (1970), que caracteriza como seguros os princípios para a área aplicada da educação, uma vez que são derivados de pesquisa experimental e possuem uma metodologia para seu emprego específico na tentativa de otimizar a habilidade de leitura. Matos (1992), ao sugerir a especificação completa do comportamento que se deseja ensinar, o reforçamento imediato dos comportamentos-objetivo, o reforçamento somente das respostas efetivamente apresentadas pelo aluno, a utilização de situações de aprendizagem naturalmente reforçadoras, a escolha cuidadosa de situações antecedentes de ensino aprendizagem e o alerta de que o erro é aversivo e produz paradas temporárias ou permanentes no comportamento, reafirma a eficácia do embasamento experimental para um ensino eficaz da habilidade de leitura, assim como Gettinger (1993) e Kohler e Greenwood (1990), ao colocarem o aumento da performance do aluno fazendo-se a correção imediata do erro e a liberação de reforçamento verbal. Também Cleary e Packham (1968) apresentaram as máquinas de ensinar baseando-se nos princípios comportamentais: um instrumental mecânico em que o feedback era imediato e as respostas corretas eram reforçadas socialmente com sons de aplausos de um auditório.

Entre os primeiros estudos publicados pelo periódico Journal of Applied Behavior Analysis (JABA) estão os de Hall, Panyan, Rabon e Broden (1968a), e Thomas, Becker e Armstrong (1968), que apresentam os conceitos propostos pela Análise do Comportamento sendo aplicados também em classes regulares pelos professores, variando a performance e aplicando reforçadores. Em seu primeiro volume, o JABA publica como primeiro artigo a pesquisa Effects of teacher attention on study behavior (Hall, Lund \& Jackson, 1968b), no qual a performance acadêmica é colocada sob controle da atenção do professor, introduzindo a aplicação dos princípios comportamentais em uma situação de sala de aula. Esses estudos colocam possibilidades da atuação do analista do comportamento na situação escolar já nos primeiros números publicados dos periódicos, sugerindo o interesse dos pesquisadores da área na educação.

Uma das maneiras de se verificar a aplicação das proposições teóricas de uma determinada abordagem é pela produção escrita sobre ela. Azzi, Oliveira e Pereira (1992), resgatando as idéias de Subramanyan, mencionam que a publicação dos trabalhos realizados cumpre as funções de servir como meio de registro e preservação dos conhecimentos construídos pelo homem, tendo como característica importante o fato de ser um registro público - qualquer pessoa pode submeter um trabalho para publicação e pode também adquiri-la; conferir prestígio e reconhecimento aos autores, aos editores, à comissão que julga os artigos para a publicação e até aos próprios assinantes; e disseminar informações, visto que foi justamente para agilizar o processo de comunicação científica que o periódico foi idealizado.

Ao se analisar a produção escrita de uma abordagem, evidenciam-se tendências, os procedimentos mais utilizados, o autor ou autores mais citados nas publicações, as áreas do conhecimento que mais contribuem para a produção. Também é possível verificar a recenticidade dos artigos ou livros que são utilizados como referências, quais artigos e/ou livros são mais utilizados e a nacionalidade dos referenciados. Possibilita, ainda, avaliar como tem sido a comunicação entre diferentes tipos de pesquisa dentro de uma mesma área - se trabalhos teóricos ou pesquisas básicas ou aplicadas são referenciadas. Portanto, a produção científica é produto também das contingências da comunidade em que é elaborada, sendo modelada e mantida por ela (Andery, Micheletto \& Sério, 1999).

Analisar a produção científica requer um trabalho de investigação. E também um tipo de produção de conhecimento, uma vez que oferece a elucidação de questões presentes nos dias de hoje e não a mera quantificação. Moura (1997) faz um alerta a esta questão:

\section{A contagem de publicações seria um passo superficial em checar a qualificação de uma comunidade científica; entretanto, por resultar em uma massa de resultados consistentes, acaba possibilitando à instituição visualizar o seu quadro de produção, detectar o seu estado-da-arte, alertá-la quanto ao grau de réplica de pesquisa e propiciar/originar novas estratégias a serem priorizadas como ciência. Uma instituição, pela avaliação de sua produção, pode alcançar parâmetros que venham embasar e permitir- the reavaliar, repensar sua missão e reprogramar suas estratégias de desenvolvimento e necessidades. (p. 11, 12)}

Portanto, a avaliação histórica da produção científica contextualiza e permite o planejamento de novas ações. A produção científica sobre análise do comportamento tem sido feita, entre outras formas, por meio de listas bibliográficas, pela análise de publicações e análise de citações (Witter, 1997). As pesquisas de revisão efetuadas por Winett e Winkler (1972), Northup, Vollmer e Serret (1993), Laties e Mace (1993), SulzerAzaroff e Gillat (1990) sugerem que os dados apresentados em revisões podem fornecer um importante contexto da direção futura da produção científica em questão. Winett e Winkler (1972) revisaram 14 números do JABA e investigaram quais os tipos de comportamento-alvo foram reforçados em classes comuns, identificando qual direção estaria sendo tomada pelos analistas do comportamento na 
educação regular. Verificaram que comportamentos classificados como inapropriados é que são escolhidos para mudança, assim como os comportamentos que mantêm o status quo da instituição ou dos sujeitos que contratam os serviços. Fizeram um alerta à comunidade de analistas do comportamento quanto à direção das pesquisas da área, revisando os artigos até então publicados.

Northup e colaboradores (1993) estabeleceram uma classificação quanto a tipos de artigos publicados: artigos de pesquisa, artigos metodológicos, artigos de revisão e discussão e outros, concluindo por uma contribuição consistente, mas pequena, dos artigos de discussão e revisão, com uma média de $12 \%$ da produção do periódico nos vinte e cinco anos de sua publicação. Essa classificação, apontando as áreas de menor produção de pesquisas, indica ao pesquisador da análise do comportamento áreas de investigação que necessitam de trabalhos.

Laties e Mace (1993), em artigo comemorativo de 25 anos de publicação do JABA, apresentam, mediante uma análise de citações presentes no periódico, a contribuição que os artigos têm oferecido a outras publicações e em uma ampla variedade de situações, desde distúrbios de desenvolvimento como déficit de aprendizagem, até as situações que interferem no funcionamento do lar e da escola. Apresentam, ainda, as tendências e direções das pesquisas publicadas, colocando a grande contribuição da Análise do Comportamento aplicada para o campo dos distúrbios de desenvolvimento, mas que existem novas fronteiras para esta área de pesquisa.

Sulzer-Azaroff e Gillat (1990), ao realizarem uma revisão histórica pretendendo organizar uma reedição de artigos do JABA, fazem uma análise de 347 artigos já publicados nesse periódico e verificam que 70 destes artigos foram indicados por especialistas da área de educação como representativos da produção científica em Análise do Comportamento, com investigações de diferentes conteúdos (conduta, treino de habilidades sociais, linguagem, etc.) e em diferentes níveis de ensino. Apresentam resultados em que a categoria "academic" (habilidades específicas do aluno como leitura, matemática, ...) está bem representada no período entre os anos 197377 , com $14 \%$, e tem acentuado declínio nos períodos subseqüentes, $1978-82$ com $6 \%$, chegando ao patamar de $5 \%$ no período de 1983-86.

Marmo (2002), analisando 214 artigos do JABA e tendo como tema básico a educação, demonstrou que as publicações na área de educação têm, em sua grande maioria, mantido a especificidade da análise aplicada do comportamento. Os artigos, metodologicamente, priorizam os delineamentos de sujeito único, sala de aula como local de realização e um maior número de trabalhos empregando técnicas de reforçamento positivo e como sujeitos-alvo das pesquisas encontram-se em maior número as que lidam com os pré-escolares.
Pesquisas sobre a habilidade acadêmica de leitura figuram desde os primeiros anos de publicação do periódico, por exemplo Cleary e Packham (1968), com a máquina de ensinar leitura, e Gray, Baker e Stancyk (1969), que apresentam um sistema chamado PDI (Performance Determined Instruction) como possibilidade de um trabalho de intervenção para treino na remediação de leitura empregado em uma clínica especializada e são de relevância social como proposto por Baer, Risley e Wolf (1968). A sociedade reconhece o que é feito, pois a leitura é necessária para a realização de qualquer atividade dentro de uma classe do ensino regular e, se colocada sob controle adequado, pode ser responsável pelo sucesso do aluno, garantindo a ele o acesso a parte do conhecimento do mundo e sendo fonte de inserção em algumas comunidades.

Considerando os artigos acima mencionados como representativos da atuação do analista do comportamento na área da educação regular, pretendeuse, nesta pesquisa, caracterizar os artigos publicados no JABA pertinentes à habilidade acadêmica de leitura em classes de ensino regular, identificando variáveis experimentais manipuladas, variáveis de observação medidas, resultados encontrados, tempo de duração dos procedimentos empregados e tipos de reforçadores utilizados, sujeitos das pesquisas, aplicadores e delineamento experimental. A hipótese é de que se possa verificar, assim, se esta habilidade está sendo ensinada na educação dita formal e como.

O comportamento de ler e escrever visto pela perspectiva da análise do comportamento, segundo Fonseca (1997),

pode auxiliar sobremaneira na elaboração de programas de
ensino na etapa de alfabetização, pois permite definir com
clareza as etapas do processo de aprendizagem de leitura e
de escrita, aspectos e seqüencias de seu ensino e
instrumentos necessários para sua aprendizagem. (p. 22)

\section{Método}

Fonte. O periódico Journal of Applied Behavior Analysis (JABA) foi o escolhido para a presente investigação, pois é o mais antigo periódico representativo da publicação em Análise do Comportamento Aplicada.

\section{Procedimento}

O procedimento definidor dos artigos selecionados para composição do banco de dados deste estudo foi estabelecido em consonância com o trabalho de Marmo (2002), que também utilizou artigos do JABA na área de educação. As palavras-chave Reading, Spelling, Reading classroom, Reading skills, Spelling skills e Spelling classroom foram lançadas na ferramenta de busca da home page do periódico e forneceram artigos relacionados à 
habilidade de leitura.

A partir dos títulos dos artigos, eliminaram-se das listas todos os artigos que promoviam intervenções com sujeitos portadores de deficiências (de hiperativos a autistas e portadores de deficiências físicas) e os artigos que tinham como comportamento-alvo outro que não o ensino ou a correção de comportamento acadêmico em sala de aula, e foram excetuados ainda, os artigos em que os sujeitos eram de jardim de infância e pré-escola. Por fim foram eliminados artigos de revisão, biografias e resenhas.

Chegou-se, dessa maneira, a um total de 36 artigos, dos quais 35 foram localizados (pois um deles era do ano de 2003 e ainda não disponível nas bibliotecasfonte). Depois de lidos na íntegra, os artigos foram distribuídos em uma planilha onde foram sistematizadas as seguintes informações: a) ano de publicação do artigo, b) filiação dos autores, c) sujeitos, d) nível de escolaridade, e) delineamento utilizado na intervenção, f) variáveis experimentais e de observação (Matos, 1990), g) setting, h) tempo total de duração da intervenção.

Tal categorização foi consensual entre duas das autoras do trabalho, buscando-se marcas da Análise do Comportamento na prática de ensino. Mediante essas informações foi possível quantificar a produção de artigos específicos sobre a habilidade de leitura no ensino regular, o número de autores e sua filiação, as possíveis interlocuções ou isolamento dos autores que investigaram esta habilidade, a regularidade das publicações, os procedimentos e objetivos dos autores, verificando nesses trabalhos possíveis aproximações ou afastamentos do que é esperado da Análise do Comportamento aplicada ao ensino de leitura em sala regular.

\section{Resultados e Discussão}

A leitura na íntegra dos 35 artigos que pareciam preencher os critérios estabelecidos para sua manutenção no banco de dados permitiu uma observação mais acurada e 15 artigos, inicialmente selecionados, foram ainda descartados quer por não tratarem da sala de aula regular e sim de ambiente dentro da escola especialmente preparado para a pesquisa, quer por tratar de pesquisa realizada com alunos que tinham outros problemas comportamentais. O banco de dados foi então fixado em 20 artigos:

1. McLaughlin, T. F. \& Malaby, J. (1972). Intrinsic reinforces in a classroom token economy. Journal of Applied Behavior Analysis, 5, 263-260.

2. Lahey, B. B., McNees, M. P. \& Brown, C. C. (1973). Modification of deficits in reading for comprehension. Journal of Applied Behavior Analysis, 6, 475-480.

3. Van Houten, R., Hill, S. \& Parsons, M. (1975). An analysis of a performance feedback, public posting, and praise upon academic performance and peer interaction.
Journal of Applied Behavior Analysis, 8, 449-457.

4. Carnine, D. W. (1976). Effects of two teacher-presentation rates on off-task behavior, answering correctly, and participation. Journal of Applied Behavior Analysis, 9, 199-206.

5. Dineen, J. P., Clark, H. B. \& Risley, T. R. (1977). Peer tutoring among elementary students: Educational benefits to the tutor. Journal of Applied Behavior Analysis, 10, 231-238.

6. Schwartz, G. J. (1977). College students as contingency managers for adolescents in a program to develop reading skills. Journal of Applied Behavior Analysis, 10, 645-655.

7. Konarski, Jr. E. A., Johnson, M. R., Crowell, C. R. \& Whitman, T. L. (1980). Response deprivation and reinforcement in applied settings: A preliminary analysis. Journal of Applied Behavior Analysis, 13, 595-609.

8. Trovato, J. \& Bucher, B. (1980). Peer tutoring with or without home-based reinforcement, for reading remediation. Journal of Applied Behavior Analysis, 13, 129-141.

9. Glover, J. A., Zimmer, J. W., Filbeck, R. W. \& Plake B. S. (1980). Effects of training students to identify the semantic base of prose materials. Journal of Applied Behavior Analysis, 13, 655-667.

10. Kirby, K. C., Holborn, S. W., Bushby, H. T. (1981). Word game bingo: A behavioral treatment package for improving textual responding to sight words. Journal of Applied Behavior Analysis, 14, 317-326.

11. Kistner, J., Hammer, D., Wolfe, D., Rothblum, E., Drabman, R. S. (1982). Teacher popularity and contrast effects in a classroom token economy. Journal of Applied Behavior Analysis, 15, 85-96.

12. Greer, R. D. \& Polirstok, S. R. (1982). Collateral gains and short-term maintenance in reading and on-task responses by inner-city adolescents as a function of their use of social reinforcement whilw tutoring. Journal of Applied Behavior Analysis, 15, 123-139.

13. Gettinger, M. (1985). Effects of teacher-directed versus student-directed instruction and cues versus no cues for improving spelling peformance. Journal of Applied Behavior Analysis, 18, 167-171.

14. Greenwood, C. R., Dinwiddie, G., Bailey, V., Carta, J. J., Dorsey, D., Kohler, F. W., Nelson, C., Rotholz, D., Schulte, D. (1987). Field replication of classwide peer tutoring. Journal of Applied Behavior Analysis, 20, 151-160.

15. Kohler, F. W. \& Geenwood, C. R. (1990). Effects of collateral peer supportive behaviors within the classwide peer tutoring program. Journal of Applied Behavior Analysis, 23, 307-322.

16. Geenwood, C. R., Terry, B., Mayer, C. A., Finney, R. (1992). The classwide peer tutoring program: Implementation factors moderating students' achievement. Journal of Applied Behavior Analysis, 25, 101-116.

17. Gettinger, M. (1993). Effects of invented spelling and direct instruction on spelling performance of secondgrade boys. Journal of Applied Behavior Analysis, 26, 281-291.

18. Belfiore, P. J., Skinner, C. H., Ferkis M. A. (1995). Effects of response and trial repetition on sight-word training for student with learning disabilities. Journal of 
Applied Behavior Analysis, 28, 347-348.

19. Gilbert, L. M., Williams, L., McLaughlin, T. F. (1996). Use of assisted reading to increase correct reading rates and decrease error rates of students with learning disabilities. Journal of Applied Bahavior Analysis, 29, 255-257.

20. Noell, G. H., Gansle, K. A., Witt, J. C., Whitmarsh, E. L., Freeland, J. T., LaFleur, L. H., Gilbertson, D. N., Northup, J. (1998). Effects of contingent reward and instruction on oral reading performance at differing levels of passage difficulty. Journal of Applied Behavior Analysis, 31, 659-663.

É, pois, muito pequeno o número de estudos sobre leitura no ambiente escolar, cabendo lembrar, entretanto, que o que se propunha era exatamente pesquisar a leitura em sala de aula do ensino regular, não se considerando outros ambientes dentro da escola, como biblioteca, sala de estudos e laboratórios.

A análise dos 20 artigos permitiu inicialmente organizá-los em três grupos, conforme os procedimentos tivessem sido aplicados pelo próprio experimentador e/ou professor da classe (12 artigos), por tutores (6 artigos) e um terceiro grupo em que era avaliada a implementação dos programas de tutoria por meio do desempenho dos tutelados (2 artigos).

No primeiro grupo verifica-se o maior número de pesquisas, distribuídas da seguinte maneira: sete pesquisas têm o experimentador como aplicador, três aproveitam o professor como aplicador e em duas o experimentador é o próprio professor da classe. $\mathrm{O}$ segundo grupo inclui procedimentos em que alunos ensinam alunos. Segundo a revisão de Greenwood, Terry, Arreaga-Mayer e Finney (1992), estes procedimentos foram extensamente estudados, com delineamento de sujeito único, entre os anos de 1981 e 1989, quando o programa de tutoria foi validado, os componentes refinados e as replicações estendidas para diferentes áreas, populações estudantis e idades. É significativo ver que este procedimento foi usado em oito das pesquisas. $\mathrm{O}$ terceiro grupo indica a preocupação com a implementação dos programas de tutoria. Pesquisadores já relataram que professores algumas vezes variam o procedimento padronizado de implementação do programa de tutoria reduzindo, por exemplo, o número de sessões por semana (Greenwood e cols., 1992), o que repercute no desempenho dos tutelados.

Tabela 1 - Variáveis experimentais relativas ao delineamento em 20 artigos do JABA sobre leitura

\begin{tabular}{l|c}
\hline & $\mathrm{f}$ \\
\hline Aplicar reforçamento positivo & 18 \\
Aplicar feedback imediato & 15 \\
Utilizar tutores & 7 \\
Aplicar punição & 4 \\
Aplicar autocorreção & 4 \\
Testar reforçadores & 3 \\
Estabelecer tempo para execução de tarefas & 3 \\
Expor publicamente resultados & 2 \\
Apresentar respostas auto-avaliadoras & 1 \\
Disponibilizar materiais (Premack) & 1 \\
\hline
\end{tabular}

Quanto às variáveis experimentais identificadas, observada a definição de Matos (1990), as Tabelas 1 e 2 mostram, respectivamente, um grupo de variáveis próprias ao delineamento e outro de variáveis voltadas ao assunto em pauta: a leitura. A análise da Tabela 1 permite ver uma marca da proposta de Skinner (1972) para a educação, destacando-se a preocupação dos analistas do comportamento em utilizar reforçadores positivos: 18 das 20 pesquisas o usaram. Com relação à ocorrência de controle aversivo, a aplicação de punição foi utilizada em quatro artigos - dois na forma de perda de fichas, um na forma de reprimenda verbal e outro na diminuição do tempo de recreio, cabendo destacar que o último deles é de 1980.

Está ainda representada como variável experimental com freqüência expressiva a aplicação de feedeback imediato - em 15 dos artigos, sendo 8 das pesquisas, os autores o aplicam tanto após erro como após acerto, entretanto, em 5 deles o feedback foi dado imediatamente após o erro. Mesmo assim, encontram-se entre os 18 que trabalham com reforçamento positivo. Aplicado em procedimentos relatados em artigos ao longo dos anos do JABA, o feedback imediato é sustentado para aumentar a produtividade acadêmica, para treinar professores a usar elogios (Van Houten, 1979). Quanto ao feedback imediato do erro, Gettinger (1985) apresenta em sua revisão pesquisas que empregam a instrução direta, aplicando, entre outros procedimentos, a correção do erro para melhorar a performance de soletrar. Já a variável experimental "feedback imediato do acerto" -, sinaliza o focar da atenção do sujeito sobre o acerto, atendendo à sugestão sete de Matos (1992): "Erros são aversivos e produzem paradas temporárias ou permanentes no comportamento." Daí a preocupação em se evitar situações aversivas que impediriam a aquisição da habilidade de leitura. 
Tabela 2 - Variáveis experimentais relativas ao conteúdo leitura em 20 artigos do JABA

\begin{tabular}{l|c}
\hline & $\mathrm{f}$ \\
\hline Apresentar modelo de leitura (palavras ou trechos) verbal ou impresso & 10 \\
Repetir corretamente palavra lida inicialmente de maneira incorreta & 10 \\
Responder perguntas ou completar trechos sobre a passagem ou palavras lidas & 8 \\
Ler previamente o texto com ou sem ajuda do gravador & 4 \\
Estabelecer tempo para execução de tarefas & 3 \\
Modificar textos e listas randomizando palavras & 3 \\
Identificar palavras que dêem sentido ao texto & 2 \\
Alterar velocidade de apresentação de palavras & 1 \\
Emparelhar palavras impressas com o modelo & 1 \\
Apresentar regras fonéticas & 1 \\
Completar tarefas de leitura & 1 \\
\hline
\end{tabular}

As demais variáveis identificadas e utilizadas em um número significativo de artigos referem-se a condições de treino do sujeito - "apresentar modelo de leitura (palavras ou trechos) verbal ou impresso" e "repetir corretamente a palavra lida inicialmente de forma incorreta". Matos (1990) também apresenta a oportunidade de repetição como condição para a aquisição de comportamento:

\section{Mostrar e instruir são maneiras poderosas de ensejar comportamento em seres humanos, desde que a pessoa esteja olhando, vendo, ouvindo e, de alguma forma, repetindo o que vê e ouve.}

Quanto à variável "responder perguntas ou completar trechos sobre passagem ou palavras lidas", refere-se à condição de ler com compreensão, ou seja, a compreensão de leitura é medida pelo número de respostas corretas que o sujeito apresenta. Também a apresentação da palavra correta após o erro e a imitação do erro do aluno antes de apresentar o modelo correto foi sugerido por Gettinger (1993) como alternativa de intervenção. Este método instrucional de pareamento do negativo-positivo teoricamente auxiliaria o estudante a focar sua atenção em como o soletrar incorreto difere do soletrar correto, mesmo que esta diferença não tenha sido apontada (p. 167). Ainda Gettinger (1993), efetuando uma revisão sobre as instruções diretas envolvidas em uma apresentação de material instrucional de leitura, refere-se ao procedimento de correção de erro como um procedimento já explorado por outros pesquisadores como Foxx \& Jones (1978), Matson, Esveldt-Dawson e Kazdin (1982) e ainda por ela mesma (Gettinger 1985), sempre como suporte para melhorar a performance de soletrar. Finalmente, cabe lembrar: entre oito recomendações de Matos (1990) para uma educação eficaz, a recomendação de número cinco - "escolher cuidadosamente as situações de ensino aprendizagem" - destaca que as discriminações são mais rápidas quando são apresentados contraexemplos, confirmando a eficácia de se utilizar o feedback imediato do erro.

Uma das dificuldades enfrentadas pelos analistas do comportamento é a utilização de reforçadores positivos de baixo custo e fácil acesso, como proposto por McLaughlin e Malaby (1972), necessários à viabilidade dos procedimentos propostos. Desta maneira, procurou-se identificar os tipos de reforçadores empregados e quais os mais utilizados.

Tabela 3 - Distribuição dos artigos por tipo de reforçador utilizado

\begin{tabular}{l|c}
\hline & $\mathrm{f}$ \\
\hline Verbais & 13 \\
Fichas & 8 \\
Pontos & 4 \\
Exposição pública & 2 \\
Dinheiro & 1 \\
Auto-reforçadores (palavras auto-elogiosas) & 1 \\
Atividade contingente & 1 \\
\hline
\end{tabular}

A leitura da Tabela 3 mostra que os reforçadores verbais ("elogios, palavras de incentivo, meneio de cabeça") foram utilizados em 13 artigos, número comparável ao Psico-USF, v. 9, n. 1, p. 59-69, Jan./Jun. 2004 dos artigos onde reforçadores como fichas e pontos poderiam ser trocados por privilégios. Cabe notar que não eram, entretanto, reforçadores excludentes. E que o 
auto-reforçamento está representado em um artigo, sendo seu emprego concomitante ao reforçamento por fichas.

Verifica-se, pois, que todos os reforçadores listados são extrínsecos à situação, mas garantidos socialmente pelo menos no início da aprendizagem (uma das recomendações de Matos, 1990) e passíveis de substituição gradual pela própria habilidade estabelecida. Os reforçadores verbais descritos, presentes na maioria dos artigos, foram também objeto de investigação específica no artigo de Kohler e Greenwood (1990), quando foi verificado o controle exercido por palavras de incentivo, mesmo quando liberadas de forma não intencional. $O$ arranjo de contingências reforçadoras possibilitou a melhoria da habilidade em questão antes que a habilidade em si ganhasse essa dimensão reforçadora.

Os diferentes desenhos que ilustram os delineamentos experimentais utilizados nos artigos analisados podem ser vistos na Tabela 4 . Os procedimentos mais freqüentes são os que utilizam o delineamento de sujeito único e a intervenção simples sem reversão. Esta constatação nos remete aos princípios básicos da pesquisa em análise do comportamento, em que cada organismo é único e, por esta razão, seu desempenho só pode ser comparável com ele mesmo.

Tabela 4 - Distribuição dos artigos por tipo de delineamento experimental

\begin{tabular}{l|c}
\hline Delineamento & $\mathrm{f}$ \\
\hline Intra-sujeito & 13 \\
AB & 8 \\
Grupo & 6 \\
Linha de base múltipla & 5 \\
ABAB & 3 \\
ABCD & 3 \\
ABA & 2 \\
ABC & 2 \\
ABACAB (ou outra ordem) & 1 \\
ABCB & 1 \\
\hline
\end{tabular}

$\mathrm{O}$ delineamento $\mathrm{AB}$ justifica-se se considerarmos a habilidade de leitura como um comportamento que depois de fortalecido não pode ser retirado ou mesmo enfraquecido pela comunidade. Quanto à distribuição dos artigos em razão do nível de escolaridade dos sujeitos, verifica-se o esperado, ou seja, grande número de pesquisas nas séries iniciais do ensino regular e, confirmando Marmo (2002), garantindo a melhoria da habilidade de leitura neste período instrucional, que é fundamental ao sucesso acadêmico.

$\mathrm{Na}$ Tabela 5 estão as variáveis de observação consideradas nos artigos analisados e é possível verificar que o espectro de dez variáveis de observação está representando as modificações observadas no comportamento dos sujeitos. Cabe ressaltar que alguns artigos destacam como objetivo a acuracidade da leitura (número de palavras soletradas corretamente), outros ainda a fluência (número de palavras soletradas). Não se faz distinção entre o soletrar e o ler, sendo entendidas como atividades complementares. Entretanto, todos os artigos têm a questão da melhoria da habilidade de leitura como objetivo principal. O maior número de artigos concentra-se na variável de observação "número de palavras soletradas corretamente" - seja para aumentar a acuracidade, seja para aumentar a fluência, ampliando o repertório já existente.

Tabela 5 - Distribuição de artigos por variável de observação

\begin{tabular}{l|c}
\hline & $f$ \\
\hline Número de palavras soletradas corretamente & 7 \\
Número de realizações de outras atividades (resistência física, idéias próprias, popularidade & 5 \\
do professor, ficar no lugar, não falar alto, sinalizar com a mão para chamar o professor) & \\
Número de respostas corretas sobre o texto lido (compreensão de leitura) & 4 \\
Velocidade de emissão de palavras/Número de palavras por minuto & 3 \\
Tempo de execução das atividades de leitura & 3 \\
Número de atividades completas de leitura & 2 \\
Número de palavras soletradas e escritas corretamente & 2 \\
Número de palavras sublinhadas corretamente/incorretamente (compreensão de leitura) & 1 \\
Número de palavras soletradas incorretamente & 1 \\
Número de respostas auto-avaliadoras positivas e negativas & 1 \\
\hline
\end{tabular}


A análise dos objetivos das pesquisas permite chegar a dois grandes grupos, como já havia sido constatado por Marmo (2002), para todo o conjunto de pesquisas em educação: um grupo (com 14 artigos) em que o objetivo é o de aumento de repertório ou da habilidade de leitura propriamente dita e um grupo ( 7 artigos) em que as pesquisas têm como objetivo testar procedimentos para aumentar repertório. Note-se que um dos artigos persegue os dois objetivos (artigo 7). Nos dois grupos foram encontradas evidências de confirmação de hipótese dos pesquisadores, entretanto é preciso ressaltar que em alguns estudos os autores mencionam níveis de observação de resultados diferentes, embora na direção da confirmação da hipótese inicial, sugerindo continuidade dos estudos propostos.

Cabe notar, finalmente, que as intervenções propostas, desenvolvidas em sua maioria nas próprias salas de aula dos sujeitos, sugerem uma simplicidade nos procedimentos, uma vez que não interferem na rotina das atividades da classe, apesar de algumas vezes serem desenvolvidas somente por alguns alunos. O número de sujeitos das pesquisas variou de 2 (dois) a 211 (duzentos e onze) elementos. As pesquisas que possuem o maior número de participantes são também as que utilizam o emprego de tutores, indicando que esta condição permite a intervenção em classes regulares onde o número de alunos é maior. Todos os sujeitos foram indicados para participação na pesquisa, quer por professores, quer em virtude de resultados de aplicação de testes padronizados de nível de leitura e classificação como portadores de atraso nesta habilidade. Isso não aconteceu apenas no caso em que os sujeitos eram universitários.

\section{Considerações Finais}

A habilidade acadêmica de leitura, no conjunto de artigos selecionados, apresenta-se como área de aplicação para a comunidade de analistas do comportamento. $\mathrm{O}$ espectro das variáveis experimentais indica múltiplas possibilidades interventivas que podem ser aplicadas em uma sala de aula do ensino regular desta habilidade acadêmica.

Na condição de treino do professor nos procedimentos operantes, é possível aumentar a performance de leitura dos alunos e os procedimentos de tutoria apresentados mostram-se como alternativa eficaz para salas numerosas, o que mais se aproxima da realidade do ensino regular. Os reforçadores utilizados, todavia, são limitados quanto ao número e o emprego maciço de reforçadores sociais verbais merece uma ressalva. Este tipo de reforçador, apesar de ser de fácil acesso, é indicativo de outros comportamentos do professor, como aceitação ou rejeição do aluno (Skinner, 1972). Como a maioria dos experimentos foi aplicada pelo pesquisador, que sabemos ser treinado para a liberação destes reforçadores em condições ótimas, esta restrição - a necessidade de treino efetivo dos aplicadores - não se evidencia na descrição dos procedimentos. Outra questão a salientar seria quanto ao reforçamento por fichas, que não é um procedimento novo e ainda é visto pelos professores como um mero sistema de troca, sendo também necessário o treino dos profissionais do ensino regular para sua utilização.

Uma alternativa a essas restrições aos reforçadores utilizados poderia ser o emprego maior de situações auto-reforçadoras como feedback imediato mediante representação de desempenho pelo próprio sujeito por meio de gráficos, desenhos ou auto-aplicação de reforçadores, implementando assim o princípio da modelagem por aproximações sucessivas ao comportamento final, garantindo dessa forma as possibilidades de acerto e conseqüente aumento na performance. $\mathrm{O}$ número de pesquisas que fazem referência à aplicação dos procedimentos em sujeitos com atraso na habilidade de leitura é expressivo quanto à possibilidade de propostas de intervenção, sendo estas para remediação ou melhoria desta habilidade. Isto é, o sujeito já passou pelo processo de aquisição da habilidade de leitura e encontra-se, então, em relação aos alunos da mesma idade ou da mesma classe escolar, em um nível abaixo do esperado. Uma das suposições de não se ter encontrado procedimentos aplicados em sujeitos ingênuos diante da aquisição da habilidade de leitura pode ser derivada do fato de tais pesquisas encontrarem-se em outro nível de escolaridade, como a pré-escola, por exemplo. Entretanto, a proposta do ensino de leitura é formalizada e regularmente exigida nas séries iniciais do ensino regular na população americana, onde se poderia pressupor a existência de clientela ingênua.

Foi visto que as pesquisas garantem a atuação do analista do comportamento nas salas do ensino regular trabalhando com a habilidade de leitura, mas cabe interrogar o porquê de um número reduzido de publicações focando esta habilidade. Esta pergunta faz-se pertinente, por terem sido encontrados nos artigos analisados no presente estudo relatos de experiências bem sucedidas de aumento do número de palavras lidas e da acuracidade de leitura empregando outros procedimentos. Cabe ainda questionar a ausência de relatos de pesquisas com equivalência de estímulos nas classes regulares, tais como o desenvolvido por de Rose, J. C., de Souza, D. G. e Hanna, E. S.(1996), que se sabe ser eficaz para o ensino de leitura, com outros sujeitos e em outros settings.

\section{Referências}

Andery, M. A., Micheletto, N., Sério, M. T. (1999). Pesquisa Histórica em Análise do Comportamento. Trabalho apresentado em mesa redonda na Reunião Anual da Sociedade Brasileira de Psicologia. Campinas-SP. 
Azzi, R. G., Oliveira, G. A. P. \& Pereira, M. A. L. (1992). Formação do pesquisador e publicações periódicas especializadas. Educação e Ensino - USF, 3(1), 19-31.

Baer, D. M., Wolf, M. M. \& Risley, T. R. (1968). Some current dimensions of applied behavior analysis. Journal of Applied Behavior Analysis, 1(1), 91-97.

Bijou, S. W. (1970). What Psychology has to Offer Education. Journal of Applied Behavior Analysis, 3, 65-71.

Cleary, A. \& Packham, D. (1968). A Touch-Detecting Teaching Machine with auditory reinforcement. Journal of Applied Behavior Analysis, 1, 341-345.

Fonseca, M. L. (1997). Diagnóstico de repertórios iniciais de leitura e escrita: uma análise baseada na concepção de relações de equivalência. (Dissertação de Mestrado). São Paulo: Universidade Federal de São Carlos.

Foxx, R. M. \& Jones, J. R. (1978). A remediation program for increasing the spelling achievement of elementary and junior high school students. Behavior Modification, 2, 211-230.

Gettinger, M. (1993). Effects of error correction on third graders' spelling. Journal of Educational Research, 87(1), 39-45.

Gray, B. B., Baker, R. D. \& Stancyk, S. E. (1969). Performance determined instruction of training in remedial reading. Journal of Applied Behavior Analysis, 2, 255-263.

Greenwood, C. R., Terry, B., Mayer, C. A., Finney, R. (1992). The classwide peer tutoring program: Implementation factors moderating students' achievement. Journal of Applied Behavior Analysis, 25, 101-116.

Hall, R. V., Lund, D. \& Jackson, D. (1968a). Effects of teacher attention on study behavior. Journal of Applied Behavior Analysis, 1, 1-12.

Hall, R. V., Panyan, M., Rabon, D. \& Broden, M. (1968b). Instructing beginning teachers in reinforcement procedures which improve classroom control. Journal of Applied Behavior Analysis, 1, 315-322.

Keller, F. S. (1983). Aprendendo a ensinar: memórias de um professor universitário. São Paulo: Edcon.

Kohler, F. W. \& Greenwood, C. R. (1990). Effects of collateral peer supportive behaviors within the classwide peer tutoring program. Journal of Applied Behavior Analysis, 23(3), 307-322.

Laties V. G., Mace C. F. (1993). Taking stock: The first 25 years of the Journal of Applied Behavior Analysis. Journal of Applied Behavior Analysis, 26, 513-525.

Luna, S. V. (2002). Contribuições de Skinner para a Educação. Em Psicologia e Educação: revendo contribuições Placco. São
Paulo: Educ/Fapesp.

Marmo, A. V. (2002). Publicações sobre Educação no Journal of Applied Behavior Analysis: uma revisão. (Dissertação de Mestrado). São Paulo: PUC.

Mathews, R. M. (1997). Editors as authors: Publication trends of articles authored by JABA editors. Journal of Applied Behavior Analysis, 30, 717-721.

Matos, M. A. (1990). Controle Experimental e controle estatístico: a filosofia do caso único na pesquisa comportamental. Ciência e Cultura, 42(8), 585-592.

Matos, M. A. (1992). Análise de contingências no aprender e no ensinar. Em Alencar, E. S. (Org.). Novas contribuições da Psicologia aos processos de ensino e aprendizagem. São Paulo: Cortez.

Matson, J. L., Esveldt-Dawson, K., Kadzin, A. E. (1982). Treatment of spelling deficits in mentally retarded children. Mental Retardation, 20, 76-81.

McLaughlin, T. F. \& Malaby, J. (1970). Intrinsic reinforces in a classroom token economy. Journal of Applied Behavior Analysis, 5, 263.

Mejias, N. P. (1973). Modificação de comportamento em situação escolar. São Paulo: EPU, Editora da Universidade de São Paulo.

Moura, E. (1997). ITA - Avaliação da produção científica (1991-1995). Em G. P. Witter (Org.). Produção Cientifica. (pp. 9-24). Campinas (SP): Átomo.

Northrup, J., Vollmer, R. \& Serrett, K. (1993). Publicaction trends in first 25 years of the Journal of Applied Behavior Analysis. Journal of Applied Behavior Analysis, 26, 527-537.

Ollendick, T. H., Matson, J. L., Dawson, K. E. \& Shapiro, E. S. (1980). Increasing spelling achievement: An analysis of treatment procedures utilizing an alternating treatment design. Journal of Applied Behavior Analysis, 13, 645-6.

O'leary, K. D. (1972). Behavior modification in the classroom: A rejoinder to winett and winkler. Journal of Applied Behavior Analysis, 5, 505.

Placco, V. M. de Souza (Org.). (2002). Psicologia \& Educação. Revendo contribuições. São Paulo: Fapesp/Educ.

Rose, J. C., Souza, D. G. \& Hanna, E. S. (1996). Teaching reading and spelling: Exclusion and stimulus equivalence. Journal of Applied Behavior Analysis, 29, 451-459.

Skinner, B. F. (1969). Ciência e comportamento bumano. São Paulo: Martins.

Skinner, B. F. (1972). Tecnologia do Ensino. São Paulo: Herder.

Skinner, B. F. (1974). Sobre o behaviorismo. São Paulo: Cultrix. 
Sulzer-Azarof, B. \& Gillat, A. (1990). Trends in behavior analysis in education. Journal of Applied Behavior Analysis, 23, 491-495.

Thomas, D. R., Becker, W. C. \& Armstrong, M. (1968). Production and elimination of disruptive classroom behavior by systematically varying teacher behavior. Journal of Applied Behavior Analysis, 1, 35-45.

Van Houten, R. (1979). The perfomance feedback system: Generalization of effects across time. Child Behavior Therapy, 1(3), 219-236.

Vargas, E., Vargas, J. (1992). Designs for excellence in education: The legacy of B. F. Skinner. Em P. Richard \& L. A. Hamerlynk. Lonmont, CO: Sopris West Inc.

Winett, R. A. \& Winkler, R. C. (1972). Current behavior modification in the classroom: Be still, be quiete, be docile. Journal of Applied Behavior Analysis, 5, 499-504.

Witter, G. P. (Org.). (1997). Produção Cientifica. Campinas: Átomo.

Recebido em março de 2004 Reformulado em maio de 2004 Aprovado em junho de 2004

Sobre as autoras:

Luiza Cristina Mauad é psicóloga, mestre em Análise do Comportamento e professora de Psicologia do Centro Universitário Universitas.

Maria do Carmo Guedes é doutora em Educação e professora da Pontifícia Universidade Católica de São Paulo nos Programas de Pós-Graduação em Psicologia Social e Análise do Comportamento.

Roberta Gurgel Azzi é psicóloga, mestre em Psicologia e doutora em Educação, professora da Faculdade de Educação da Unicamp em cursos de graduação e pós-graduação. 
